Research Paper

\title{
Outpatient acupuncture effects on patient self-reported symptoms in oncology care: a retrospective analysis
}

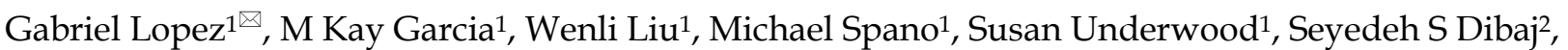 \\ Yisheng $\mathrm{Li}^{2}$, Rocio Moguel${ }^{1}$, Janet Williams ${ }^{1}$, Eduardo Bruera ${ }^{1}$, Lorenzo Cohen ${ }^{1}$ \\ 1. Department of Palliative, Rehabilitation, and Integrative Medicine, The University of Texas MD Anderson Cancer Center \\ 2. Department of Biostatistics, The University of Texas MD Anderson Cancer Center \\ $\square$ Corresponding author: gabriel.lopez@mdanderson.org \\ (C) Ivyspring International Publisher. This is an open access article distributed under the terms of the Creative Commons Attribution (CC BY-NC) license \\ (https://creativecommons.org/licenses/by-nc/4.0/). See http://ivyspring.com/terms for full terms and conditions.
}

Received: 2018.04.06; Accepted: 2018.08.05; Published: 2018.09.08

\begin{abstract}
Background: Increased access to complementary therapies such as acupuncture at academic medical centers has created new opportunities for management of cancer and cancer treatment related symptoms.

Methods: Patients presenting for acupuncture treatment during calendar year 2016 at an outpatient integrative medicine clinic in a comprehensive cancer center were asked to complete a modified Edmonton Symptom Assessment Scale (ESAS; 16 symptoms, score 0-10, 10 worst possible) before and after each visit. ESAS subscales analyzed included global (GDS; score 0-90), physical (PHS, 0-60) and psychological distress (PSS, 0-20). ESAS symptom score change pre/post acupuncture treatment \& from baseline visit to first follow up were evaluated by paired t-test.

Results: Of 375 participants [mean age 55.6, 68.3\% female, 73.9\% white, most common cancer diagnosis of breast (32.8\%) and thoracic/head \& neck (25.9\%)], $73.3 \%$ had at least one follow up acupuncture treatment [mean 4.6 (SD 5.1) treatments]. Highest/worst symptoms at baseline were poor sleep (3.92), fatigue (3.43), well-being (3.31), and pain (3.29). Statistically significant reduction/improvement (pre/post) was observed for all ESAS symptoms and subscales for the initial acupuncture treatment $(p<0.001)$. Hot flashes had the highest mean reduction (-1.93), followed by fatigue (-1.72), numbness/tingling (-1.70), and nausea (-1.67). Clinically significant reductions were also observed for ESAS subscales of GDS (-12.2), PHS (-8.5), and PSS (-2.6). For symptom change from initial acupuncture treatment to first follow up (pre/pre), statistically and clinically significant improvement was observed for spiritual pain (-1.10; $p<0.001)$ and ESAS subscale of GDS $(-6.09 ; p=0.048)$. Clinical response rates (reduction $\geq 1)$ on follow up were highest for symptoms of spiritual pain (58.9\%), dry mouth $(57.8 \%)$ and nausea $(57.3 \%)$.

Conclusions: Outpatient acupuncture was associated with immediate \& longitudinal significant improvement across a range of symptoms commonly experienced by individuals during cancer care. Further research is needed to better understand frequency of treatments needed in clinical practice to help maintain benefit.
\end{abstract}

Key words: Integrative Medicine, Integrative Oncology, Acupuncture, Complementary health approach, Patient Reported Outcomes, Edmonton Symptom Assessment System

\section{Introduction}

There has recently been increased interest in identifying non-pharmacologic interventions for improving symptom control during and after cancer care. Symptoms may develop as a result of the cancer or its treatment, with some symptoms persisting long after treatment is complete. Complementary therapies such as acupuncture may be well suited to provide symptomatic relief, alone or in conjunction with other symptom control approaches including pharmaceuticals. According to the National Center for Complementary and Integrative Health (NCCIH), acupuncture falls under the category of mind and 
body complementary health approaches. A recent review of National Cancer Institute-designated comprehensive cancer centers identified acupuncture and massage $(73.3 \%$ each) as the most frequently available integrative therapies onsite. ${ }^{1}$ With increased availability of complementary health approaches such as acupuncture in western medical settings, there is also an increased interest in learning more about the immediate and long term benefits of such interventions on symptom control and quality of life. Insight into the effects of complementary health approaches in a real-world setting may help inform clinical decision making beyond what is already known in the literature through controlled trial settings.

At the MD Anderson Cancer Center, our Integrative Medicine Center offers acupuncture treatments to cancer patients together with conventional care. Patients referred to the Integrative Medicine Center are evaluated by a physician with the goal of developing an integrative oncology treatment plan that may include referral to additional clinical services such as acupuncture, massage, nutrition, physical therapy, and/or health psychology. A part of an effort to document real-world effects, we have previously reported on the effects of an integrative oncology physician consultation on patient self-reported symptoms and quality of life. ${ }^{2}$ Integrative oncology refers to "a patient-centered, evidence-informed field of cancer care that utilizes mind and body practices, natural products, and/or lifestyle modifications from different traditions alongside conventional cancer treatments. Integrative oncology aims to optimize health, quality of life, and clinical outcomes across the cancer care continuum and to empower people to prevent cancer and become active participants before, during, and beyond cancer treatment." 3 Our current interest is to expand on current knowledge about the real-world effects of acupuncture, an integrative oncology clinical service, in an oncology care setting.

Acupuncture is an intervention that includes the placement of stainless-steel needles in specific locations throughout the body to help provide relief for one or more symptoms. It has been used as part of medical systems such as traditional Chinese medicine for thousands of years. As an intervention, acupuncture is considered safe with the most common non-serious adverse events including needling pain, hematoma/bleeding, and orthostatic symptoms. ${ }^{4}$ A number of studies have been completed demonstrating the benefits of acupuncture in helping to provide relief for cancer-related symptoms. In a systematic review of randomized-controlled trials using acupuncture by
Garcia et al, the greatest benefits were achieved for relief of symptoms including pain and nausea/vomiting. ${ }^{5}$ Data recently presented from a SWOG phase III clinical trial revealed benefits of acupuncture for relief of aromatase inhibitor induced join pain and stiffness in post-menopausal women receiving treatment for hormone sensitive breast cancer. ${ }^{6}$ We have previously reported on our experience with offering acupuncture treatments in an inpatient setting, with clinically significant improvement in symptoms of pain, sleep disturbance, anxiety, nausea, and drowsiness. ${ }^{7}$ Although numerous clinical trials have demonstrated a benefit of acupuncture in improving patient symptoms and quality of life during and after cancer care, these findings do not necessarily translate into changes observed in a real-world clinical setting.

Although there is evidence of the beneficial effects of acupuncture for symptom control in clinical trial settings, little is known about the effects of acupuncture when implemented alongside conventional cancer care in an outpatient setting. Our current study examined the immediate pre/post treatment and longitudinal/multiple treatment effects of acupuncture on patient self-reported symptoms in an outpatient clinical setting at a comprehensive cancer center.

\section{Methods}

Patients were referred to the Integrative Medicine Center from within our institution for evaluation by a physician. Subsequent referral to acupuncture was made based on physician judgement after review of a patient's oncology care history and symptom profile. A physician or advanced practice provider order from within our center was required to schedule an initial acupuncture treatment. Acupuncture was available as a fee-for-service, with reimbursement depending on individual insurance plan coverage. Those patients demonstrating financial need had access to acupuncture via available philanthropic funds. As acupuncture treatments were delivered as part of the standard of care within our clinical center; there was no control group.

Prior to each acupuncture treatment, the acupuncturist reviewed the patient's medical history, chief complaint/reason for referral, lab-work, and concurrent medications as per safety guidelines established by our program. As part of the standard of care, patients were asked to complete the Edmonton Symptom Assessment Scale (ESAS) on an electronic tablet immediately prior to and after the acupuncture visit. The acupuncturist had access to review the symptom profile prior to the intervention to help in developing a personalized treatment plan. As part of 
an IRB approved protocol, patient reported outcomes data were analyzed for the period January 1, 2016 to Dec 31, 2016.

\section{Outcome Measures}

Patient symptom burden was assessed using a modified version of the ESAS including 10 core symptoms (pain, fatigue, nausea, depression, anxiety, drowsiness, loss of appetite, decreased sense of well-being, shortness of breath, and sleep) and an additional 6 items of spiritual distress, financial distress, numbness/tingling, hot flashes, dry mouth, and memory. ${ }^{8,9}$ Symptoms were scored on a numeric scale of 0 to $10(10=$ worst possible experience of that symptom). ESAS subscales analyzed included Global Distress (GDS, 0-90, pain, fatigue, nausea, drowsiness, appetite, shortness of breath, anxiety, depression, and well-being), Physical Distress (PHS, 0-60, pain, fatigue, nausea, drowsiness, appetite, and shortness of breath), and Psychological Distress (PSS, 0-20, anxiety and depression). The ESAS has been validated in oncology care settings, with minimally clinically important differences constituting an important way to interpret patient reported outcome scores and determine the clinical effectiveness of an intervention. 10,11 An individual symptom score reduction of $\geq 1$ is considered a clinically significant change; for the ESAS subscales, reduction of GDS $\geq 3$, PHS $\geq 2$, and $P S S \geq 2$ indicates clinically significant changes.

\section{Intervention}

Acupuncture treatment was provided in a clinical space within our outpatient center by licensed, experienced $(>10$ years) staff acupuncturists credentialed through the institution's Medical Staff Office. During an acupuncture treatment, small, sterile, stainless steel needles, typically ranging from 32-40 gauge, were inserted into specific acupuncture points on the body and left in place for 20-30 minutes. The needle's effects may have been augmented with manual or electrical stimulation and/or heat. The length and frequency of acupuncture treatments varied according to the condition being treated per professional judgement of the acupuncturist. Typically, two treatments weekly for 3-4 weeks, are initially recommended, but treatment plans can vary according to the symptoms being addressed. Treatments may then decrease in frequency as the treatment plan progresses and desired results are achieved. Initial acupuncture visit duration was approximately one hour, with follow up visits lasting approximately $30-45$ minutes.

\section{Statistics}

Demographics, baseline ESAS individual symptoms and subscales are summarized by descriptive statistics which include frequencies and proportions for categorical and mean +/- standard deviation and median (range) for continuous variables. Change in pre/post ESAS symptoms and subscales for those who have a symptom score at baseline of 1 or greater was evaluated by paired t-test. Pre/Post acupuncture treatment ESAS data availability was reported for all patients and for those with a baseline score of at least 1 . Completion rate was calculated for pre and post treatment symptoms scores. ESAS completion is defined as completing $80 \%$ or more of the questionnaire (13 out of 16 questions). The change in ESAS symptoms and subscales pre-session from baseline to first visit was evaluated by paired t-test for those patients who had their first follow-up visit within 30 days of baseline. Response was defined as any reduction in symptom score (greater or equal to 1 point). Response rate was calculated and reported along with a Clopper-Pearson (exact) confidence interval for patients who had a baseline score of greater or equal to 1 and had at least one follow-up visit within 30 days. The association of demographics and baseline ESAS symptoms with number of follow-up visits ( 0 vs $>=1$ ) was evaluated by chi-square/Fisher's exact test for categorical variables and Wilcoxon rank sum test for continuous variables. Next, pre-treatment symptom score change from baseline to the last treatment (i.e. pre-treatment last session - pre-treatment first session) was compared between patients who had total of 4 or 5 treatments with those who 6 or more treatments. The association of response rate and follow-up status (no follow-up vs at least 1 follow-up) was evaluated by chi-square (or Fisher's exact) test. The probability of 'at least one follow-up visit' was evaluated for association with response to treatment by applying a logistic regression model. Estimates of odds ratio and its $95 \%$ confidence interval are reported.

\section{Results}

We examined data for 375 unique patients receiving acupuncture treatment between January and December 2016, with a mean number of acupuncture treatments of 4.6 (range: 1-48, SD 5.1) for a total of 1728 treatments during the time period [Table 1]. Pre-treatment ESAS data were available for $352 / 375(93.9 \%)$ at the baseline encounter; pre- and post-treatment ESAS data were available for 253/375 $(68.3 \%)$ of the initial/baseline encounters, with 275 patients (73.3\%) having at least one follow up visit. The mean number of days from initial consult to first follow up was 10 (SD 20.3) days, with 259/375 (69\%) having their first follow up treatment within 30 days. Patient characteristics included mean age of 55.6, mostly female (68.3\%), white/caucasian (73.9\%), 
married or with significant other $(76 \%)$, and living locally $(49.6 \%)$. Most common cancer types included breast, thoracic/head and neck, and gastrointestinal. The distribution of marital status and residency location is different between patients who had at least one follow-up visit and those who had no follow-ups.

Table 1: Patient demographics and clinical characteristics at initial consult and first follow up.

\begin{tabular}{|c|c|c|c|c|}
\hline & & $\begin{array}{l}\text { Initial Consult } \\
(n=375)\end{array}$ & $\begin{array}{l}\text { Follow Up } \\
(\mathrm{n}=275)\end{array}$ & p-value \\
\hline \multirow[t]{2}{*}{ Age } & Mean \pm Std & $55.6 \pm 13.1$ & $56.3 \pm 12.4$ & 0.18 \\
\hline & Median (Min, Max) & $57.0(8.0,88.0)$ & $\begin{array}{l}57.0(8.0, \\
88.0)\end{array}$ & \\
\hline \multirow[t]{2}{*}{ Gender } & Female & $256(68.3 \%)$ & $191(69.5 \%)$ & 0.41 \\
\hline & Male & $119(31.7 \%)$ & $84(30.5 \%)$ & \\
\hline \multirow[t]{6}{*}{ Race } & White/Caucasian & $277(73.9 \%)$ & $205(74.5 \%)$ & 0.45 \\
\hline & Hispanic/Latino & $42(11.2 \%)$ & $32(11.6 \%)$ & \\
\hline & Black/African American & $22(5.9 \%)$ & $13(4.7 \%)$ & \\
\hline & Asian & $20(5.3 \%)$ & $16(5.8 \%)$ & \\
\hline & Other & $6(1.6 \%)$ & $3(1.1 \%)$ & \\
\hline & Unknown & $8(2.1 \%)$ & $6(2.2 \%)$ & \\
\hline \multirow[t]{5}{*}{ Marital Status } & $\begin{array}{l}\text { Married/Significant } \\
\text { Other }\end{array}$ & $285(76.0 \%)$ & $218(79.3 \%)$ & 0.035 \\
\hline & Single & $46(12.3 \%)$ & $29(10.5 \%)$ & \\
\hline & Divorced & $32(8.5 \%)$ & $18(6.5 \%)$ & \\
\hline & Widowed & $9(2.4 \%)$ & $8(2.9 \%)$ & \\
\hline & Unknown & $3(0.8 \%)$ & $2(0.7 \%)$ & \\
\hline \multirow[t]{5}{*}{ Residency } & Harris County & $133(35.5 \%)$ & $114(41.5 \%)$ & $<0.001$ \\
\hline & $\begin{array}{l}\text { Seven Surrounding } \\
\text { Counties }\end{array}$ & $53(14.1 \%)$ & $39(14.2 \%)$ & \\
\hline & Rest of Texas & $65(17.3 \%)$ & $40(14.5 \%)$ & \\
\hline & Rest of US & $106(28.3 \%)$ & $71(25.8 \%)$ & \\
\hline & International & $18(4.8 \%)$ & $11(4.0 \%)$ & \\
\hline \multirow[t]{10}{*}{ Cancer Type } & Breast & $123(32.8 \%)$ & $92(33.5 \%)$ & 0.32 \\
\hline & Thoracic/Head and Neck & $97(25.9 \%)$ & $72(26.2 \%)$ & \\
\hline & Gastrointestinal & $57(15.2 \%)$ & $37(13.5 \%)$ & \\
\hline & Lymphoma/Myeloma & $33(8.8 \%)$ & $21(7.6 \%)$ & \\
\hline & Genitourinary & $24(6.4 \%)$ & $18(6.5 \%)$ & \\
\hline & Gynecologic & $17(4.5 \%)$ & $14(5.1 \%)$ & \\
\hline & Leukemia & $9(2.4 \%)$ & $7(2.6 \%)$ & \\
\hline & No evidence of disease & $6(1.6 \%)$ & $6(2.2 \%)$ & \\
\hline & Skin & $5(1.3 \%)$ & $4(1.5 \%)$ & \\
\hline & Other & $4(1.1 \%)$ & $4(1.5 \%)$ & \\
\hline \multirow{2}{*}{$\begin{array}{l}\text { Number of } \\
\text { treatments }\end{array}$} & Mean \pm Std & $4.61 \pm 5.12$ & & \\
\hline & Median (Min, Max) & $3.0(1.0,48.0)$ & & \\
\hline \multirow{2}{*}{$\begin{array}{l}\text { Days to } 1^{\text {st }} \\
\text { follow-up }\end{array}$} & Mean \pm Std & $10.06 \pm 20.34$ & & \\
\hline & Median (Min, Max) & $5.0(1.0,193.0)$ & & \\
\hline
\end{tabular}

"Represents comparison between those did not follow up $(n=100)$ and those with at least one follow up $(n=275)$. Significant difference is represented in bold.

\section{Baseline symptoms at initial acupuncture treatment}

The most frequently reported symptoms on initial presentation included sleep $(81.3 \%)$, well-being $(79.2 \%)$, and fatigue $(77.6 \%)$. The highest self-reported symptom scores on initial presentation were: sleep 3.9 (SD 2.7), fatigue 3.4 (SD 2.6), well-being 3.3 (SD 2.3), pain 3.3 (SD 2.7), and poor memory 3 (SD 2.4) [Table 2]. Moderate intensity symptom scores (4-7) were observed for symptoms of sleep (4.5), numbness tingling (4.5), hot flash (4.4), fatigue (4.2), pain (4.1) and dry mouth (4).

Table 2. Baseline mean symptom scores and single treatment symptom score change.

\begin{tabular}{|c|c|c|c|c|c|c|}
\hline ESAS Symptoms & $\begin{array}{l}\text { All Patients } \\
\text { treated } \\
\text { Mean (SD) }\end{array}$ & $\mathbf{n}^{*}$ & $\begin{array}{l}\text { Pre } \\
\text { Mean } \\
\text { (SD) }\end{array}$ & $\begin{array}{l}\text { Post } \\
\text { Mean (SD) }\end{array}$ & $\begin{array}{l}\text { Mean } \\
\text { change }^{* *}\end{array}$ & de \\
\hline Pain & $3.29(2.66)$ & 280 & $4.13(2.32)$ & $2.07(2.16)$ & $-1.57(1.69)$ & $<.001$ \\
\hline Fatigue & $3.43(2.55)$ & 291 & $4.15(2.20)$ & $2.13(2.06)$ & $-1.72(1.82)$ & $<.001$ \\
\hline Nausea & $0.90(1.80)$ & 112 & $2.82(2.18)$ & $0.40(0.96)$ & $-1.67(2.10)$ & $<.001$ \\
\hline Shortness of Breath & $0.94(1.70)$ & 116 & $2.86(1.82)$ & $0.50(1.27)$ & $-1.55(1.54)$ & $<.001$ \\
\hline Appetite & $2.68(2.64)$ & 237 & $3.98(2.26)$ & $2.07(2.27)$ & $-1.01(2.09)$ & $<.001$ \\
\hline Drowsiness & $1.85(2.19)$ & 210 & $3.10(2.05)$ & $1.48(1.85)$ & $-0.97(1.90)$ & $<.001$ \\
\hline Depression & $1.30(1.95)$ & 149 & 3.07 (1.87) & 0.90 (1.55) & $-1.14(1.40)$ & $<.001$ \\
\hline Anxiety & $2.03(2.23)$ & 221 & $3.23(2.01)$ & $1.14(1.65)$ & $-1.42(1.71)$ & $<.001$ \\
\hline Sleep & $3.92(2.68)$ & 305 & $4.52(2.36)$ & $3.16(2.52)$ & $-1.06(2.08)$ & $<.001$ \\
\hline Well-being & $3.31(2.30)$ & 297 & $3.90(1.99)$ & $2.39(2.07)$ & $-1.16(1.81)$ & $<.001$ \\
\hline Spiritual Pain & $0.89(1.69)$ & 107 & $2.91(1.86)$ & $0.59(1.37)$ & $-1.11(1.63)$ & $<.001$ \\
\hline Financial Distress & $1.84(2.61)$ & 171 & $3.78(2.58)$ & $1.73(2.53)$ & $-0.69(1.59)$ & $<.001$ \\
\hline Hot flashes & $1.91(2.8)$ & 151 & $4.42(2.65)$ & $1.13(2.11)$ & $-1.93(2.26)$ & $<.001$ \\
\hline Numbness/tinging & $2.89(2.99)$ & 226 & $4.48(2.59)$ & $1.89(2.34)$ & $-1.70(1.90)$ & $<.001$ \\
\hline Dry mouth & $2.24(2.69)$ & 197 & $4.01(2.41)$ & $1.63(2.21)$ & $-1.18(1.76)$ & $<.001$ \\
\hline Memory & $3.06(2.39)$ & 290 & $3.71(2.13)$ & $2.51(2.18)$ & $-0.83(1.57)$ & $<.001$ \\
\hline GDS 1 & $\begin{array}{l}19.72 \\
(13.09)\end{array}$ & 34 & $\begin{array}{l}34.91 \\
(13.06)\end{array}$ & $\begin{array}{l}13.01 \\
(10.17)\end{array}$ & $\begin{array}{l}-12.18 \\
(10.28)\end{array}$ & $<.001$ \\
\hline PHS 1 & $13.09(8.9)$ & 45 & $\begin{array}{l}22.82 \\
(8.66)\end{array}$ & $8.57(6.76)$ & $-8.46(6.42)$ & $<.001$ \\
\hline PS & 75) & 136 & 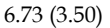 & $2.03(2.91)$ & $-2.57(2.89)$ & 5.00 \\
\hline
\end{tabular}

* Patients with individual symptom score of at least 1 on the pre-treatment ESAS.

For ESAS subscale calculation, all symptoms in subscale must have score of at least 1 to be included.

** For all symptoms, a change score of $\geq 1$ is considered clinically significant. For predetermined subscales, clinical significance is defined as follows: $\geq 3$ for GDS; $\geq 2$ for PHS and PSS. Clinically significant change in bold.

${ }^{1}$ GDS (Global distress score) equals sum of pain, fatigue, nausea, depression, anxiety, drowsiness, appetite, well-being, and shortness of breath (total score 0-90); PHS (physical distress score) equals sum of pain, fatigue, nausea, drowsiness, appetite, and shortness of breath (total 0-60); and PSS (psychological distress score) equals sum of depression and anxiety.

\section{Immediate pre-post effects of acupuncture treatment}

Immediate pre-post effects of acupuncture treatment were significant across all symptoms and subscales $(p<0.001)$. Clinically significant effects were observed for symptoms of anxiety (-1.42), appetite $(-1.01)$, depression (-1.14), dry mouth (-1.18), fatigue $(-1.72)$, hot flash (-1.93), nausea (-1.67), numbness/tingling (-1.70), pain (-1.57), shortness of breath (-1.55), sleep (-1.06), spiritual pain (-1.11), and well-being (-1.16). ESAS subscale scores of GDS, PHS, and PSS also showed clinically significant improvement.

\section{Longitudinal effects of acupuncture treatment}

Of the 259 patients who had their first acupuncture follow-up visit within 30 days from the baseline, $215(83.0 \%)$ completed pre-treatment ESAS measures at their first visit. Of this sample, pre-treatment measures at both acupuncture sessions 
(initial and follow-up) were available for 209 (80.7\%) patients. Statistically significant improvement between the baseline (pre-initial treatment) and follow-up (pre-follow up treatment) ESAS scores was observed for all symptoms except appetite [Table 3]. Both statistically and clinically significant improvement was observed for the symptom of spiritual pain $(-1.10 ; \mathrm{p}<0.001)$. Although we observed statistically significant improvement for ESAS subscales of GDS and PSS, these remained clinically significant only for GDS. Clinical response rates were greater than $50 \%$ for symptoms of nausea, shortness of breath, well-being, spiritual pain, hot flashes, numbness/tingling, and dry mouth [Table 3]. On evaluation of the association of response to the first treatment and likelihood of follow-up, there was only a statistically significant association between hot flashes and follow-up $(\mathrm{p}=0.014)$. The odds of having at least one follow-up is 3.3 times higher for those who did not respond to their first treatment with regards to hot flashes compared to those who did respond (data not shown).

Since most treatment plans included the recommendation for 1 to 2 acupuncture treatments per week for 4 weeks, we also analyzed overall symptom score change from pre-treatment at baseline to the pre-treatment at the last session in patients who had 4 or more treatments within a 30 days period $(n=137 ; 38 \%)$. We compared the population of patients with a total of 4 or 5 acupuncture treatments $(n=68)$ with those who had at least 6 treatments $(n=69)$. Our analyses do not indicate any statistically significant association between number of treatments (4 or 5 versus 6 or more) and pre-treatment symptom score change from the first to the last treatment (data not shown).

\section{Discussion}

Our results can serve an important role in the design of future clinical trials as well as helping to inform real-world clinical practice. A recently published National Cancer Institute monograph based on a symposium "Acupuncture for Cancer Symptom Management," identified as a future direction the need to advance the evidence-based integration of acupuncture into conventional cancer care settings. ${ }^{12}$ Areas of practice improvement highlighted included exploration of acupuncture effects on symptoms clusters, performing pragmatic trials to evaluate effectiveness of acupuncture as part of usual care, and identifying patient-reported outcomes of relevance in oncology acupuncture practice.

Table 3. Symptom score change between first treatment and first follow up.

\begin{tabular}{|c|c|c|c|c|c|c|c|}
\hline ESAS Symptoms & $\mathrm{n}^{*}$ & $\begin{array}{l}\text { Initial } \\
\text { Mean (SD) }\end{array}$ & $\begin{array}{l}\text { Follow Up } \\
\text { Mean (SD) }\end{array}$ & Mean change ${ }^{* *}$ & p-value & $\mathrm{n}+$ & Clinical response rates + \\
\hline Pain & 205 & $4.07(2.33)$ & $2.98(2.41)$ & $-0.53(2.17)$ & $<.001$ & 197 & $47.2 \%(39.9 \%, 54.5 \%)$ \\
\hline Fatigue & 216 & $4.10(2.14)$ & $3.11(2.49)$ & $-0.50(2.26)$ & 0.004 & 205 & $44.5 \%(37.5 \%, 51.7 \%)$ \\
\hline Nausea & 78 & $2.55(1.93)$ & $0.84(1.67)$ & $-0.56(1.87)$ & 0.011 & 76 & $57.3 \%(45.4 \%, 68.7 \%)$ \\
\hline Shortness of Breath & 79 & $2.85(1.80)$ & $0.77(1.52)$ & $-0.78(2.00)$ & 0.001 & 75 & $50.7 \%(38.7 \%, 62.6 \%)$ \\
\hline Appetite & 169 & $4.04(2.23)$ & $2.63(2.65)$ & $-0.34(2.68)$ & 0.112 & 162 & $48.4 \%(40.4 \% \%, 56.5 \%)$ \\
\hline Drowsiness & 151 & $2.98(1.95)$ & $1.72(1.99)$ & $-0.44(1.93)$ & 0.007 & 147 & $48.2 \%(38.8 \%, 55.7 \%)$ \\
\hline Depression & 108 & $2.94(1.79)$ & $1.19(1.84)$ & $-0.51(1.72)$ & 0.003 & 104 & $40.6 \%(30.9 \%, 50.8 \%)$ \\
\hline Anxiety & 162 & $3.08(1.91)$ & $1.76(2.08)$ & $-0.53(1.96)$ & 0.001 & 151 & $48.6 \%(40.3 \%, 57.0 \%)$ \\
\hline Sleep & 225 & $4.47(2.33)$ & $3.56(2.49)$ & $-0.57(2.27)$ & $<.001$ & 213 & $46.8 \%(39.8 \%, 53.9 \%)$ \\
\hline Well-being & 217 & $3.95(1.96)$ & $3.04(2.37)$ & $-0.57(1.86)$ & $<.001$ & 205 & $53.0 \%(45.8 \%, 60.1 \%)$ \\
\hline Spiritual Pain & 78 & $2.97(1.85)$ & $0.69(1.53)$ & $-1.10(2.13)$ & $<.001$ & 75 & $58.9 \%(46.8 \%, 70.3 \%)$ \\
\hline Financial Distress & 122 & $3.63(2.48)$ & $1.52(2.39)$ & $-0.54(1.17)$ & 0.007 & 116 & $49.1 \%(39.5 \%, 58.7 \%)$ \\
\hline Hot flashes & 103 & $4.39(2.68)$ & $1.74(2.64)$ & $-0.78(2.15)$ & $<.001$ & 97 & $55.9 \%(45.2 \%, 66.2 \%)$ \\
\hline Numbness / tinging & 165 & $4.55(3.02)$ & $2.57(2.79)$ & $-0.75(2.02)$ & $<.001$ & 154 & $52.4 \%(44.0 \%, 60.6 \%)$ \\
\hline Dry mouth & 150 & $4.22(2.43)$ & $2.08(2.50)$ & $-0.73(2.02)$ & $<.001$ & 140 & $57.8 \%(49.0 \%, 66.2 \%)$ \\
\hline Memory & 209 & $3.61(2.10)$ & $2.63(2.31)$ & $-0.43(1.63)$ & $<.001$ & 196 & $47.6 \%(40.4 \%, 55.0 \%)$ \\
\hline GDS1 & 24 & $34.54(12.80)$ & $18.04(12.70)$ & $-6.09(13.96)$ & 0.048 & 24 & $47.8 \%(26.8 \%, 69.4 \%)$ \\
\hline PHS1 & 32 & $22.48(8.77)$ & $12.05(8.51)$ & $-3.23(9.37)$ & 0.069 & 31 & $50.0 \%(31.3 \%, 68.7 \%)$ \\
\hline PSS1 & 95 & $6.41(3.26)$ & $2.96(3.61)$ & $-0.84(3.40)$ & 0.023 & 91 & $33.0 \%(23.3 \%, 43.8 \%)$ \\
\hline
\end{tabular}

* Patients with at least one follow up treatment and with an individual symptom score of at least 1 on the pre-treatment ESAS. For ESAS subscale calculation, all symptoms in subscale must have score of at least 1 to be included.

* For all individual symptoms, a change score of $\geq 1$ is considered clinically significant. For predetermined subscales, clinical significance is defined as follows: $\geq 3$ for GDS; $\geq 2$ for PHS and PSS. Clinically significant change in bold.

+ Patients with first follow up treatment within 30 days and with an individual symptom score of at least 1 on the initial visit, pre-treatment ESAS. All symptoms in subscale must have score of at least 1 to be included in the response rate analysis.

\# Clinical response rate (95\% confidence interval) defined as a 1-point decrease or more in score on the ESAS individual symptoms; 2-point decrease or more in score on the ESAS PSS; 3-point decrease or more in score on the ESAS PHS and GDS.

${ }^{1}$ GDS (Global distress score) equals sum of pain, fatigue, nausea, depression, anxiety, drowsiness, appetite, well-being, and shortness of breath (total score 0-90); PHS (physical distress score) equals sum of pain, fatigue, nausea, drowsiness, appetite, and shortness of breath (total 0-60); and PSS (psychological distress score) equals sum of depression and anxiety. 
Our goals were to learn about the effects of acupuncture on self-reported symptoms when offered in a clinical setting alongside conventional oncology care. We observed statistically and clinically significant reduction/improvement in most individual ESAS symptoms and across all subscales after a single acupuncture treatment. At first follow up, we also observed statistically significant improvement across most symptoms surveyed, with clinically significant improvement in the individual symptom of spiritual pain and global and physical distress subscales. Clinical response rates were near or above $50 \%$ for the majority of individual symptoms analyzed. We also learned about the feasibility of collecting immediate pre/post and longitudinal self-reported outcome data as part of routine clinical practice in an integrative medicine center offering acupuncture and other complementary health approaches, with pre-treatment patient reported outcome data available in greater than $90 \%$ of encounters and both pre/post treatment data available in greater than $65 \%$ of encounters.

A strength of this study is that it mimics current clinical practice for acupuncture. Acupuncture treatments were based on the best clinical judgement of acupuncturists in our clinical center, not on a standardized treatment protocol. There are several limitations, however. First, although these findings provide important information for the design of future trials, lack of a control group is a recognized limitation; thus, results should be interpreted with caution. Second, as with any study involving patient-reported symptom management, biologic measures were unavailable. Finally, the patient population is from a single integrative medicine clinic at a comprehensive cancer center, which may not be representative of the population of patients receiving acupuncture treatments in the community during their cancer care. As part of a real-world study, patients were referred to acupuncture treatments as part of the standard of care on the basis of clinical recommendation by a physician and / or advanced practice provider. Although we limited our analysis of symptom score change between initial treatment and first follow up to those treatments scheduled within 30 days, it is possible that other medical interventions (e.g., concurrent acupuncture in the community) or changes in disease course could be responsible for the changes observed.

Our findings related to clinically significant symptom score change, clinical response rates, and dose effect can help inform future studies exploring effects of acupuncture when offered in a real-life clinical setting alongside conventional cancer care. We have also learned about the feasibility of collecting patient reported outcomes as part of the standard of care in an outpatient integrative oncology program at a comprehensive cancer center. Our results suggest the potential benefit of acupuncture treatments in providing clinically significant improvements for commonly reported cancer related-symptoms during cancer care. With increased interest in the non-pharmacologic management of symptoms such as pain in the supportive care of cancer patients, ${ }^{13,14}$ more research is needed to better understand acupuncture mechanisms, dosing, and its effects on self-reported symptoms.

\section{Acknowledgements}

This research study was supported in part by funding from the Duncan Family Institute for Cancer Prevention and Risk Assessment.

\section{Competing Interests}

The authors have declared that no competing interest exists.

\section{References}

1. Yun H, Sun L, Mao JJ. Growth of Integrative Medicine at Leading Cancer Centers Between 2009 and 2016: A Systematic Analysis of NCI Designated Comprehensive Cancer Center Websites. J Natl Cancer Inst Monogr. 2017 Nov 1;2017(52).

2. Lopez G, McQuade J, Cohen L, et al. Integrative Oncology Physician Consulations at a Comprehensive Cancer Center: Analysis of Demographic, Clinical and Patient Reported Outcomes. J Cancer 2017; 8(3): 395-402.

3. Witt CM, Balneavues LG, Cardoso MJ, et al. A comprehensive definition for integrative oncology. J Natl Cancer Inst Monogr. 2017 Nov $1 ; 2017(52)$.

4. Melchart D, Weidenhammer W, Streng A, et al. Prospective investigation of adverse effects of acupuncture in 97733 patients. Arch Intern Med. 2004;164:1104-105.

5. Garcia MK, McQuade J, Haddad R, et al. Systematic review of acupuncture in cancer care: as synthesis of the evidence. J Clin Oncol. 2013 Mar 1;31(7):952-60.

6. Hershman DL, Unger JM, Greenlee H, et al. Randomized blinded shamand waitlist-controlled trial of acupuncture for joint symptoms related to aromatase inhibitors in women with early stage breast cancer (S1200). GS4-04. San Antonio Breast Cancer Symposium, Dec 72017.

7. Garcia MK, Cohen L, Spano M, et al. Inpatient acupuncture at a major cancer center. Integr Cancer Ther. 2018 Mar;17(1):148-152. doi: 10.1177/1534735416685403. Epub 2017 Jan 4.

8. Bruera E, et al. The Edmonton Symptom Assessment System (ESAS): a simple method for the assessment of palliative care patients. J Palliat Care 1991;7:6.

9. Delgado-Guay MO, Chisholm G, Williams J, et al. Frequency, intensity, and correlates of spiritual pain in advanced cancer patients assessed in a supportive/palliative care clinic. Palliat Support Care. 2016 Aug;14(4):341-8.

10. Hui D, Shamieh O, Eduardo Palva C, et al. Minimal Clinically Important Differences in the Edmonton Symptom Assessment Scale in Cancer Patients: A Prospective, Multicenter Study. Cancer. 2015 Sep 1;121 (17):3027-35.

11. Hui D, Shamieh O, Paiva CE, et al. Minimally Clinically Important Difference in Physical, Emotional, and Total Symptom Distress Scores of the Edmonton Symptom Assessment System. Journal of Pain and Symptom Management. Vol 1; No 2. Feb 2016. 262-269.

12. Zia FZ, Oluwadamilola $\mathrm{O}, \mathrm{Bao} \mathrm{T}$, et al. The National Cancer Institute's Conference on Acupuncture for Symptom Management in Oncology: State of the Science, Evidence, and Research Gaps. JNCI Monographs, Vol 2017, Issue 52, 1 Nov 2017.

13. Tick H, Nielsen A, Pelletier KR, et al. Evidence-Based Nonpharmacologic Strategies for Comprehensive Pain Care: the Consortium Pain Task Force White Paper. Explore 2018; 14:177-211. 
14. Lyman GH, Greenlee H, Bohlke K, et al. Integrative Therapies During and After Breast Cancer Treatment: ASCO Endorsement of the SIO Clinical Practice Guideline. J Clin Oncol. 2018 Jun 11:JCO2018792721. doi: 10.1200/JCO.2018.79.2721. [Epub ahead of print] 